\title{
E-textiles prototypes and applications on wearable devices
}

\begin{abstract}
This scientific research has the purpose of studying conductive textiles, also known as "smart" textiles. At the ending of the study, we performed the assembly of functional prototypes to give the technology proof of concept to further studies. Between these prototypes are the "smart" jacket, the "smart" pillow, and the "smart" t-shirt. All of these prototypes have electronic devices integrated with conductive textile fabrics and yarns. The functionality of the prototypes involves the obtained vital signals from the user's body, such as heartbeat, and identification of the "touch" made by the user's hand to send commands to a computer or a cellphone screen.
\end{abstract}

Volume 7 Issue 5 - 2021

\author{
Jéssica Maia Piccirillo,' Renato Giacomini² \\ 'Department of Electrical Engineering, University FEI, Brazil \\ ${ }^{2}$ Department of Textile Engineering, University FEl, Brazil
}

Correspondence: Renato Giacomini, Department of Textile Engineering, University FEI, Brazil, Email renato@fei.edu.br

Received: October 02, 202। | Published: October 21, 202 I

\section{Introduction}

As stated in ${ }^{1}$ As the industry matures and develops, there is an increasing drive to turn research results into commercial opportunities. The surge in wearable technology is acting as a spur to the smart textile industry, and smart garments are being perceived as a niche market within the fast-growing wearable technology industry. The Smart Fabrics Conference 2014 has been renamed Smart Fabrics and Wearable Technology, Intel has launched a "Wearable" challenge, and "wearable tech" has never been more talked about in the media.

According to, ${ }^{2}$ the global smart fabrics market size was valued at USD 878.9 million in 2018 and is anticipated to expand at a CAGR of $30.4 \%$ from 2019 to 2025 . Smart textiles/fabrics are advanced fabrics developed with novel technologies, which offer added value to the wearer. Some of these fabrics also accumulate energy from the surroundings by extracting vibrations, heat, or sound, responding to these inputs. Such textiles are used across several applications to enhance performance and add aesthetic value. For instance, in the fashion industry, they are used due to their ability to light up and change color.

Active smart textiles are textiles that can sense the stimuli from the environment and respond to that particular stimulus. ${ }^{3}$ This can be achieved by integrating an actuator function and a sensing device. An example is a temperature-aware shirt which will automatically roll up its sleeves when body temperature becomes elevated. Very smart or intelligent textiles: textiles that can sense the environmental stimuli, give reaction to the stimuli, and thirdly adapt their behavior to the given circumstances. In the future, intelligent fabrics are expected to be integrated with cloud computing. For example, patients with a homecare medical device could send vital signals to their doctor to diagnose their health condition.

The main challenge for the devices sensible to the "smart" textiles, according to ${ }^{4}$ is their compatibility with the skin of the user, especially with his needs of comfort characteristics. The inclusion of conductive yarns and electronic devices should be minimal as possible to maintain comfort in the fabric. Consequently, the interface circuits have to deal with the fragility of the signals and the noise.

In order to interconnect different components such as sensors, batteries or processing circuits, conductive tracks have to be embedded into/onto the fabric, as they would lie on a classic motherboard. Electrically conductive fabrics need to be soft and comfortable to wear, rather than hard and rigid. There are mainly four ways to create tracks in/on textile: weaving, knitting, embroidery of conductive threads, and patterning (or coating) of conductive inks. In the first two processes, interconnection network is created during textile manufacturing process, whereas embroidery and patterning processes occur at later stage on finished fabrics. These four integration methods imply various strains in the conductive material as well as various electrical performances that should be considered. ${ }^{5}$

According to ${ }^{6}$ the smart textiles had been developed with new technologies that provide additional value to clothing. E-Textiles are known as smart clothes, electrical textiles, and smart fabric. Smart fabrics could be aesthetic or functional. The aesthetic category includes fabrics that turn on lights and can also change their colors. Meanwhile, the functional fabrics are made to be used in athletics, extreme sports, and military applications. Making the rigid electronic boards in malleable smart wearables is not an easy task.

In this research, we are trying to improve ways of capturing and emitting signals with the conductive fabric, using sewn conductive yarns and conductive dyeing fabrics in our laboratory tests. One of the most respectful universities in the world Massachusetts Institute of Technology already researched the technology of the "smart" textiles, a current publication in their website is evidence of this trend. According to, ${ }^{7}$ researchers at MIT have now embedded highspeed optoelectronic semiconductor devices, including light- emitting diodes (LEDs) and diode photodetectors, within fibers that were then woven at Inman Mills, in South Carolina, into soft, washable fabrics and made into communication systems. This marks the achievement of a long-sought goal of creating "smart" fabrics by incorporating semiconductor devices - the key ingredient of modern electronics - which until now was the missing piece for making fabrics with sophisticated functionality.

To do a complete study of the conductive textiles this would be the sequence of this study:

1. Manufacturing of the "smart" jacket using conductive yarns and electronic devices (microcontrollers, batteries, and so on)

2. Mapping of the electronic circuit and Oscilloscope test

3. Analysis of the results of the Oscilloscope test

4. Assembly of the "smart" pillow and connection with the electronic components 
5. Manufacturing of the "smart" t-shirt using conductive yarns, sensors such as humidity sensors, microcontrollers, and an app specially designed for these applications

6. Tests on using a different type of conductive textile with conductive dyeing to test conductive properties in different conductive textiles for incentive further research to explore these materials to see the impact in the user's comfort.

7. Conclusions of the tests and suggestions for further studies.

\section{Prototypes description and results}

\section{"Smart" jacket}

For the manufacturing of the "smart" jacket, we sewed one jacket already made, after that, we included conductive yarns on the jacket wrist electronics components, were connected with these conductive yarns (as the circuit indicated in the image below), such as the battery, microcontroller that are in the accessory "tag". This accessory will allow the user to send the commands made with the "smart" fabric through the data to send by Bluetooth, controlled by the jacket wrist (Figure 1).

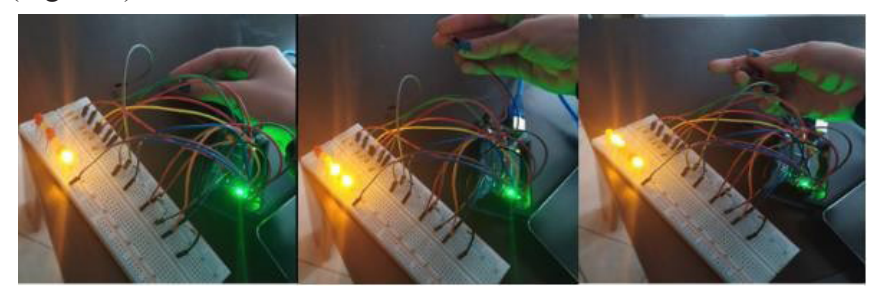

Figure I Flexible sensor programming test.

The conductive yarns were sewn by hand in a square geometry, this four-sided design allowed the structure to become a flexible sensor in the fabric. The malleable sensor with the electrical components can identify electrical signals from the user's forefinger.

The tests made in the Lab of Electrical Engineering of Fei measured the magnetically electric signals identified by our malleable sensors and a battery (Figure 2).

Resistor 100 $\Omega$

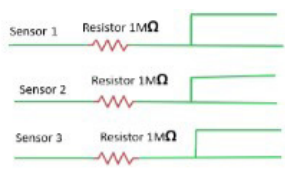

Figure 2 Electronic circuit.

It's possible to observe the behavior of the electrical signal when they interact with the user's forefinger, in the oscilloscope. When one malleable sensor is pressured by the user's finger, occurs a change in the capacitance. This change creates a delay in the electrical signal (simple model RC of an electrical circuit). The "amplitude" of this delay is a measure of the intensity of the "touch" made by the user (Figure 3).

\section{Oscilloscope test}

The tests performed are illustrated in the picture below. The data obtained through the oscilloscope is evident in the graph. The relationship between areas compressed by the user's fingers in the conductive textile and the length amplitude of the wave can be observed in the chart below.

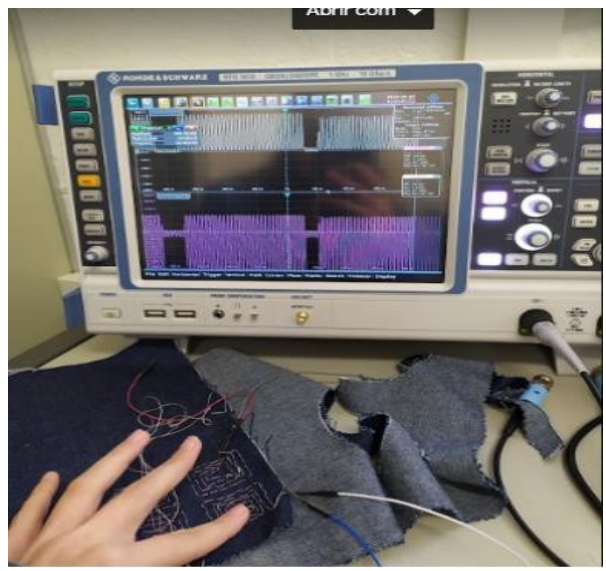

Figure 3 Oscilloscope test.

The "smart" pillow was manufactured with cotton yarns in the warp and composite copper/polymeric yarns in the weft. The composite yarns gave the textile the necessary characteristics to conduct electricity (Figure 4).

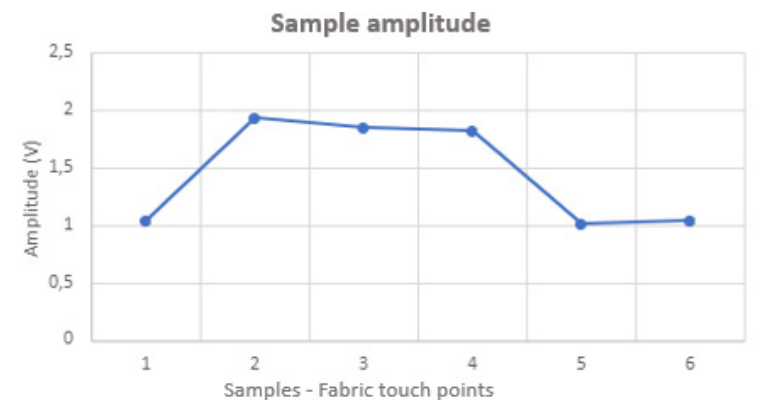

Figure 4 Amplitude versus Compressed areas in the conductive textile.

The prototype "smart" pillow uses the same electronic circuit and the same sensing method used in the "smart" jacket. The difference is that the "smart" pillow was manufactured in a weaving machine, creating a different pattern between weft and warp. Nevertheless, the correlation between conductive textiles and electronics is very similar. The "smart" pillow was created with the purpose of a potential integration with a "smart" house, an adornment that could send the user's commands to other electronic systems as a cellphone, a TV, and so on (Figure 5).

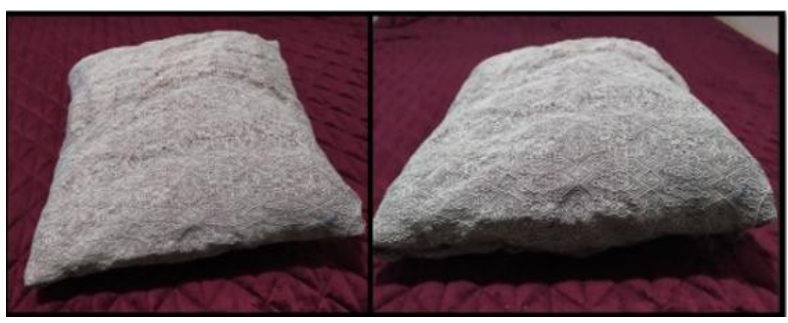

Figure 5 "Smart" pillow.

When manufacturing the "smart" $t$-shirt, malleable sensors made of conductive yarns, similarly to the previous prototypes, were included in a regular t-shirt. However, differently from the other prototypes, we utilized the Lilypad Arduino instead of Field Programmable Gate Arrays (FPGA) and the Arduino Uno. Lilypad Arduino, it's smaller if compared to the Arduino Uno and has openings to hand sewing. The conductive yarns were sewn in the Lilypad Arduino openings, and the other electronic components (Bluetooth, batteries, and so on) 
were also easily integrated. At the end of the process, the "smart" t-shirt could obtain information about the user, such as temperature and body moisture. The programming code programmed the Lilypad to send signals for an app specially developed for this prototype. The app was developed using the MIT App Inventor 2 platform, studied in the General Electronic EL100 class at University Center of FEI (Figure 6).

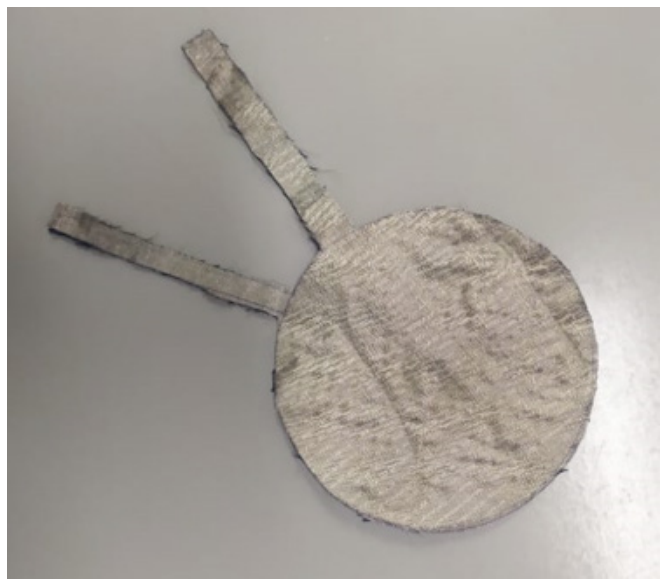

Figure 6 Shieldex bremen RS sample.

One concern about the integration of conductive textiles in garments is the aspects of comfort for the user. Because of this concern, we decided to do secondary tests, using a different type of conductive textile with conductive dyeing. We utilized Shieldex Bremen RS [ref] in those tests.

The tests were made using an Arduino code and one battery to identify if the Shieldex Bremen RS could react to the user's touch. We also tested how the Shieldex Bremen RS respond to the pressure of different materials such as a pen (Figure 7). ${ }^{8-10}$

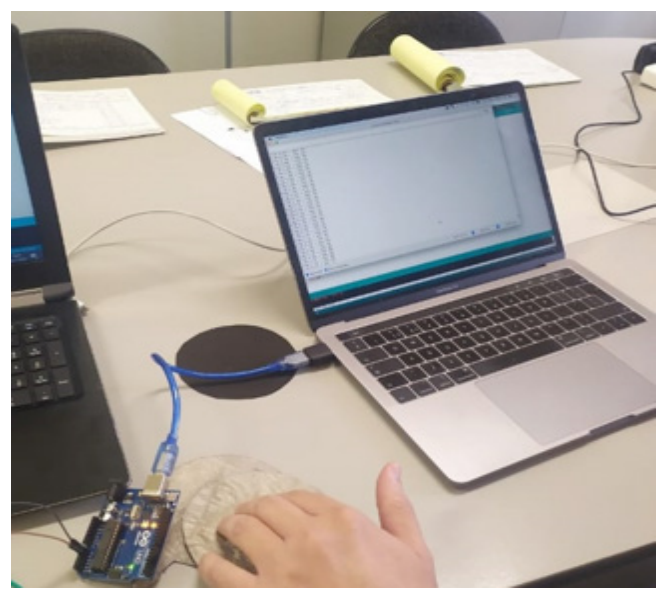

Figure 7 Shieldex bremen RS tests.

\section{Conclusion}

We can conclude that e-textiles have the potential to be implemented in the garments of the future. One can conclude that the conductivity of electricity through the copper yarns and conductive dyeing textiles and their integration with electronic components for interaction with the user is feasible. The prototypes were able to execute the functionality according to the commands sent. The malleable sensors could respond to the pressure made by the user's forefingers. Using the Shieldex Bremen RS, we made the code respond only to the user's pressure on the fabric, and not react when other objects such as a pen made the same pressure. Nevertheless, it's highly recommended that further research attempt to investigate the comfort of the conductive fabrics and other ways of having an electrical signal with higher precision.

\section{Acknowledgments}

None.

\section{Funding}

None.

\section{Conflicts of interest}

The authors declare there is no conflict of interest in publishing the article.

\section{References}

1. APS Ohmatex, Dalsgaard Christian, Sterrett Rachael. Market opportunities for smart textiles 2014 white paper on smart textile garments and devices: a market overview of smart textile wearable technologies. 2021.

2. Smart fabrics market size, share. Industry analysis report, 2019-2025. Grandviewresearch.com. 2021.

3. Nigusse Abreha Bayrau, Mengistie Desalegn Alemu, Malengier Benny, et al. Wearable smart textiles for long-term electrocardiography Monitoring—a review. Sensors. 2021;21(12):4174.

4. Lara OD. A survey on human activity recognition using wearable sensors. IEEE Communications surveys \& Tutorials, 2013.

5. De Mulatier Séverine, Nasreldin Mohamed, Delattre Roger, et al. Electronic circuits integration in textiles for data processing in wearable technologies. Advanced Materials Technologies. 2018;3(10):1700320.

6. Pessoa PS, Giacomini R. Improved controller for a large area tactile sensor based on fabric. University Center of FEI, São Bernardo do Campo.

7. Chandler DL. Introducing the latest in textiles: Soft hardware. 2019.

8. Tarafder N. Applications of wearable electronics as smart clothing. Hooghly Engineering and Technology, Hooghly. 2018.

9. Konow. Textile electrodes in electromyography measurements for hand gesture recognition. Aalto.fi, 2020.

10. Instructables. Z Patch: Hybrid Resistive/Capacitive E Textile Input. Instructables. 2021. 